\title{
Asymptotic Expansions for Regularization Methods of Linear Fully Implicit Differential-Algebraic Equations
}

\author{
M. Hanke
}

Caused by a regrettable error of the editors, in the above paper on p. 521 two figures were missing. The corresponding passage has to be read as follows:

Example: Both parametrizations (2.14) and (2.20) have a nice interpretation for some differential-algebraic equations describing electrical networks. Consider the electrical circuit of Figure 1.

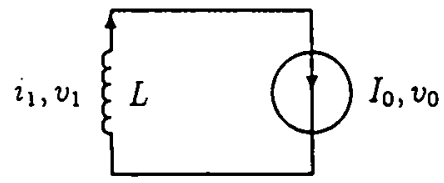

Figure 1

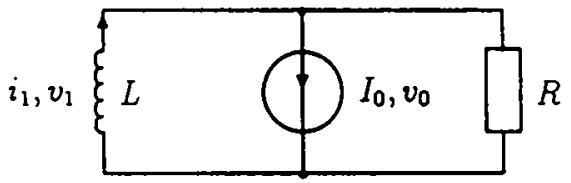

Figure 2

The circuit equations are

$$
\begin{aligned}
i_{1}^{\prime} & =-L^{-1} v_{0} \\
0 & =i_{1}-I_{0} .
\end{aligned}
$$

Taking into account the inner resistance of the current source, a better model would be the circuit given in Figure 2 with a large $R$. Now, the equations read

$$
\begin{aligned}
i_{1}^{\prime} & =-L^{-1} v_{0} \\
0 & =i_{1}+L R^{-1} i_{1}^{\prime}-I_{0} .
\end{aligned}
$$

Letting $R=\varepsilon^{-1} L$ we just obtain (2.20). The parametrization (2.14) arises if, additionally, the inductivity $L$ is perturbed by a factor $1+\varepsilon$.

M. Hanke: Humboldt-Universität zu Berlin, FB Math., PSF 1297, D - 10099 Berlin 